\title{
El análisis de redes en Iberoamérica: una agenda de investigación
}

\author{
Molina, José Luis ${ }^{1}$; Teves, Laura²; Maya Jariego, Isidro ${ }^{3}$
}

\section{Resumen}

El análisis de redes sociales ha prosperado de forma extraordinaria en el mundo Iberoamericano en los últimos años. En esta contribución se describe un estado del desarrollo de los estudios empíricos actuales, se presenta un grupo destacado de contribuciones a la IV Mesa Hispana en Portorož y se propone una agenda de investigación en torno a la discusión del concepto de mesoestructura, como alternativa para el estudio de una variedad de temas sustantivos y como un enfoque que permite avanzar en el desarrollo de la teoría social.

\section{Seis años después ...}

Antes de la Conferencia Internacional celebrada en Sitges en 1998 el análisis de redes sociales era un tema conocido por solamente unos pocos especialistas universitarios en España, México, Chile, Portugal, normalmente por haber sido estudiantes de sociología en Estados Unidos o Canadá o por haber realizado estancias en esas Universidades. La influencia de Groningen (Holanda) y Lille (Francia) todavía no se había dejado sentir, a pesar que Frans Stockman y Tom Snjders formaron parte activa de la organización del Congreso. Seis años después nos encontramos con una realidad bien distinta: 300 usuarios de la lista REDES, la existencia de una Revista electrónica ya consolidada, la aparición de números monográficos (Araucaria) y compilaciones de artículos clásicos traducidos al castellano, la presencia del ARS en cursos de doctorado, seminarios, jornadas, el acceso fácil a materiales de formación y programas informáticos, etcétera. Esta IV Mesa Hispana es un buen ejemplo de este éxito: 11 comunicaciones presenciales, muchas de ellas presentadas en inglés en la Conferencia

\footnotetext{
${ }^{1}$ Universitat Autònoma de Barcelona joseluis.molina@uab.es.

2 Universidad Nacional de La Plata Iteves@fcnym.unlp.edu.ar

${ }^{3}$ Universidad de Sevilla isidromj@us.es
} 
ordinaria; 10 comunicaciones no presenciales y 7 comunicaciones de estudios en curso.

De nuevo, la pregunta: ¿qué es lo que ha pasado? Como siempre tenemos que recordar que el análisis de redes sociales debe parte de su éxito a un equívoco, o si se quiere plantear en positivo, a su polisemia. No es ningún pecado. Otras expresiones célebres, como la de capital social sin ir más lejos, comparten esta característica. Quizás en Ciencias Sociales y Humanidades, los conceptos fuertes son forzosamente polisémicos, lo cual les dota de una capacidad heurística innegable al tiempo que les resta capacidad analítica (estamos hablando de cultura, personalidad, comunidad, etcétera). Sea como fuere, las redes sociales, como metáfora, como paradigma o como técnica, siguen en el ojo del huracán.

La segunda explicación de este éxito se debe sin duda a los sociogramas. Aunque los que trabajamos con redes los utilizamos para ilustrar más que para analizar y explicar, éstos confieren un atractivo y una credibilidad que nos sobrepasa. iQué le vamos a hacer!

Una tercera explicación está sin duda en la incorporación de académicos e investigadores procedentes de las ciencias "duras", los cuales han contribuido a aplicar el ARS con éxito a grandes cantidades de datos, como la red de Internet, el correo electrónico de organizaciones, las redes eléctricas de alta tensión... y el descubrimiento de leyes como la del mundo pequeño. De las aplicaciones militares y policiales, mejor no hablar: nosotros, en tanto que académicos, investigadores o consultores, solamente podemos pretender beneficiar a las poblaciones con las que trabajamos, ya sean colectivos de inmigrantes o trabajadores de una empresa. Nosotros publicamos nuestros datos de forma libre $y$ trasparente $y$, es bueno recordarlo, en la mayor parte de los casos nos guían intereses puramente intelectuales.

Pero hay una cuarta explicación, a nuestro juicio la más profunda y de más largo alcance: probablemente la aproximación de redes sociales permite afrontar las dicotomías clásicas en Ciencias Sociales de una forma diferente; permite avanzar en teoría social, en la forma en la que representamos el 
mundo. En esta cuarta explicación nos detendremos brevemente en esta ponencia. Pero antes, veamos cuál es la composición del ARS iberoamericano.

\section{EI ARS en Iberoamérica}

El éxito al que hacíamos referencia hace un momento puede verse plasmado en la evolución de las visitas al web REDES y a la Revista REDES en los últimos 11 meses:

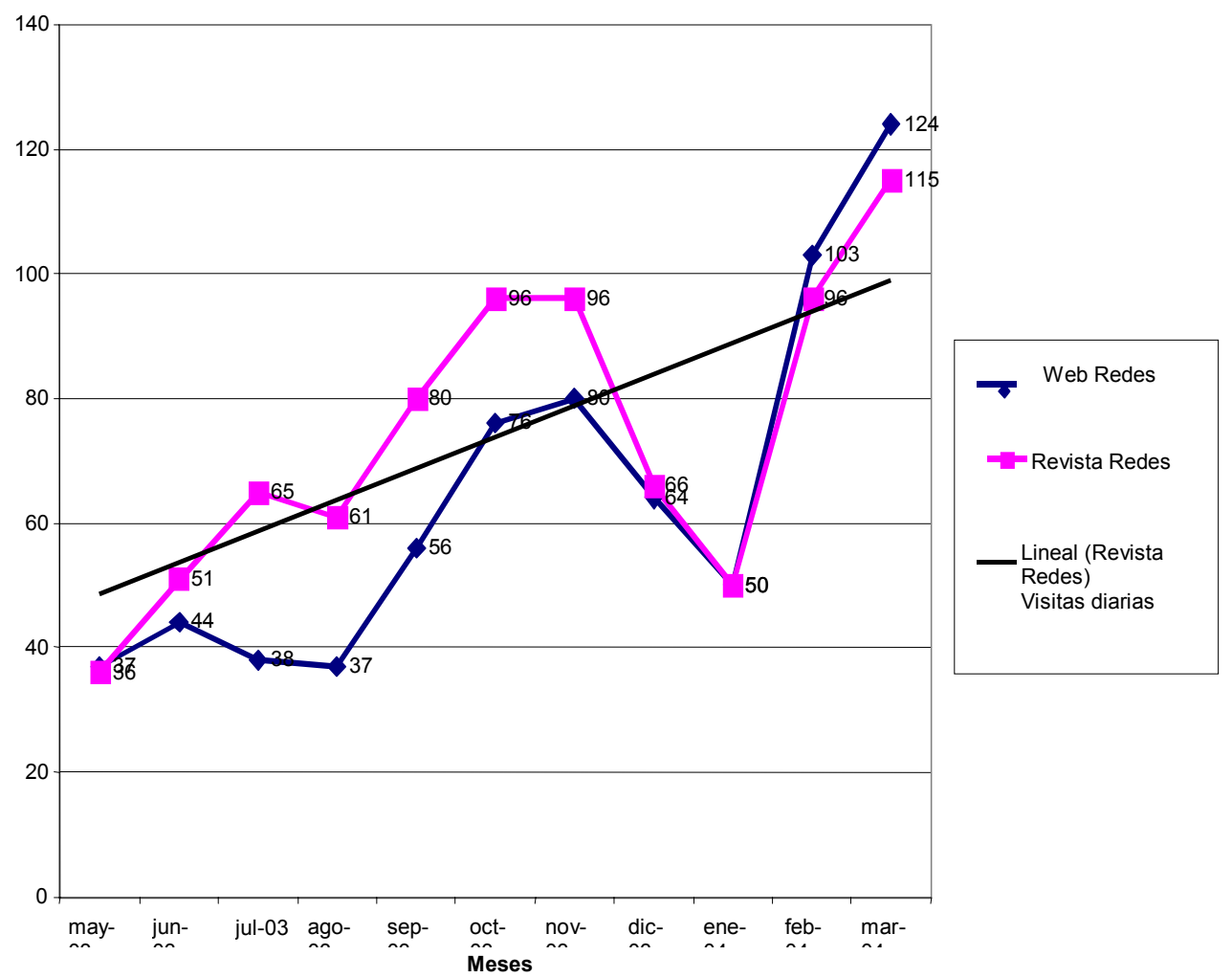

Ilustración 1. Evolución de las visitas diarias en los últimos 11 meses

El crecimiento de los usuarios de la lista REDES ha sido más lento, con etapas de estancamiento. De los 300 usuarios dados de alta a día de hoy (Abril 2004), la distribución por dominios es la siguiente: 


\begin{tabular}{|c|c|c|c|c|c|}
\hline \multicolumn{6}{|c|}{ Dominio } \\
\hline & & Frecuencia & Porcentaje & $\begin{array}{l}\text { Porcentaje } \\
\text { válido }\end{array}$ & $\begin{array}{l}\text { Porcentaje } \\
\text { acumulado }\end{array}$ \\
\hline \multirow[t]{26}{*}{ Válidos } & ES & 79 & 26,3 & 26,3 & 26,3 \\
\hline & $\mathrm{COM}$ & 74 & 24,7 & 24,7 & 51,0 \\
\hline & AR & 27 & 9,0 & 9,0 & 60,0 \\
\hline & $\mathrm{MX}$ & 22 & 7,3 & 7,3 & 67,3 \\
\hline & NET & 21 & 7,0 & 7,0 & 74,3 \\
\hline & $\mathrm{CO}$ & 12 & 4,0 & 4,0 & 78,3 \\
\hline & $\mathrm{CL}$ & 11 & 3,7 & 3,7 & 82,0 \\
\hline & BR & 9 & 3,0 & 3,0 & 85,0 \\
\hline & EDU & 9 & 3,0 & 3,0 & 88,0 \\
\hline & ORG & 8 & 2,7 & 2,7 & 90,7 \\
\hline & PE & 6 & 2,0 & 2,0 & 92,7 \\
\hline & $\mathrm{CU}$ & 3 & 1,0 & 1,0 & 93,7 \\
\hline & FR & 3 & 1,0 & 1,0 & 94,7 \\
\hline & PT & 3 & 1,0 & 1,0 & 95,7 \\
\hline & $\mathrm{NL}$ & 2 & ,7 & ,7 & 96,3 \\
\hline & UY & 2 & ,7 & ,7 & 97,0 \\
\hline & $\mathrm{CA}$ & 1 & ,3 & , 3 & 97,3 \\
\hline & CR & 1 & , 3 & ,3 & 97,7 \\
\hline & DE & 1 & ,3 & ,3 & 98,0 \\
\hline & EC & 1 & ,3 & ,3 & 98,3 \\
\hline & $\mathrm{HU}$ & 1 & ,3 & ,3 & 98,7 \\
\hline & IL & 1 & ,3 & ,3 & 99,0 \\
\hline & INFO & 1 & ,3 & ,3 & 99,3 \\
\hline & $\mathrm{NI}$ & 1 & ,3 & ,3 & 99,7 \\
\hline & PY & 1 & ,3 & ,3 & 100,0 \\
\hline & Total & 300 & 100,0 & 100,0 & \\
\hline
\end{tabular}

Tabla 1. Distribución por dominios de las direcciones electrónicas de la lista REDES (Abril 2004)

Como puede observarse, por países España (ES) es con diferencia el que tiene más direcciones (un 26\%) seguida por Argentina ( $A R$, con un 9\%) y México $(\mathrm{MX}$, con un $7,3 \%)$ y con un amplio número de países a continuación, especialmente de Iberoamérica: $\mathrm{CO}$, Colombia, CL, Chile, BR, Brasil, PE, Perú, CU, Cuba, FR, Francia, PT, Portugal ... Evidentemente, las direcciones COM no tienen porqué distribuirse de forma homogénea entre los diferentes países, por lo que el ranking de cada país podría alterarse si se pudiese asignar cada dirección electrónica de forma unívoca a un país. Las repeticiones son, por otra parte, despreciables (4 en total).

Por tipo de dominio, y a pesar que en algunos casos la asignación ha sido problemática, los porcentajes obtenidos son los siguientes: 
Tipo_dominio

\begin{tabular}{|c|c|c|c|c|c|}
\hline & & Frecuencia & Porcentaje & $\begin{array}{l}\text { Porcentaje } \\
\text { válido }\end{array}$ & $\begin{array}{l}\text { Porcentaje } \\
\text { acumulado }\end{array}$ \\
\hline \multirow[t]{7}{*}{ Válidos } & Comercial & 154 & 51,3 & 51,3 & $\overline{51,3}$ \\
\hline & $\begin{array}{l}\text { Universidad-Centro } \\
\text { Investigación }\end{array}$ & 87 & 29,0 & 29,0 & 80,3 \\
\hline & Empresa & 27 & 9,0 & 9,0 & 89,3 \\
\hline & ONG & 18 & 6,0 & 6,0 & 95,3 \\
\hline & Administración Pública & 8 & 2,7 & 2,7 & 98,0 \\
\hline & Otros & 6 & 2,0 & 2,0 & 100,0 \\
\hline & Total & 300 & 100,0 & 100,0 & \\
\hline
\end{tabular}

Tabla 2. Frecuencias por tipo de dominio

El dominio "Comercial", pertenece de nuevo a las mensajerías tipo Hotmail o a otros proveedores de servicios. Si descontamos este concepto vemos cómo el sector universitario es el más representado, con presencias significativas de empresas, ONGs y Administraciones públicas de diversos países.

Aunque evidentemente no todos los que utilizan ARS en sus investigaciones están en la lista REDES, podemos caracterizar la situación actual en los siguientes términos: se trata de un grupo de académicos (de diferentes disciplinas), principalmente españoles, que colaboran con académicos y/o investigadores de Iberoamérica, principalmente México y Argentina, con una amplia audiencia de personas interesadas de ámbitos y sectores muy diversos. Podríamos decir que la voluntad de divulgación con la que nació la lista gracias al apoyo de Steve Borgatti y la listserv del Boston College inicialmente y la RedIris después, está plenamente cubierta. Esta colaboración, como prueba este mismo trabajo, no ha hecho más que empezar.

Paralelamente a esta activa vida electrónica, la institucionalización del ARS ha avanzado mucho en los últimos años. Se han ganado plazas universitarias, se han formado grupos de investigación y por lo que se refiere a la investigación en España, el ARS figura como uno de los objetivos prioritarios del programa nacional de I+D 2003-2006. No hay ninguna razón para suponer que esta tendencia vaya a cambiar bruscamente. Por el contrario, es de esperar que con el aumento de la densidad de 
colaboraciones iberoamericanas y con la comunidad internacional en general, las iniciativas y contribuciones que incorporen el ARS se incrementen al menos en la misma medida en los próximos años.

Qué mejor que las ponencias presentadas en esta Mesa Hispana $<$ http://revista-redes.rediris.es/webredes/ivmesahispana.htm> para tener una visión de cuál es la situación en la actualidad. En la siguiente tabla se presentan las comunicaciones, presenciales o no, por el país de origen del primer autor.

\begin{tabular}{|c|c|}
\hline País de origen & N \\
\hline AR & 7 \\
\hline ES & 7 \\
\hline MX & 4 \\
\hline CL & 3 \\
\hline CO & 2 \\
\hline FR & 1 \\
\hline HU & 1 \\
\hline VE & 1 \\
\hline CU & 1 \\
\hline PA & 1 \\
\hline Total & $\mathbf{2 8}$ \\
\hline
\end{tabular}

Tabla 3. Comunicaciones por País de origen del primer autor

Por lo que a temáticas se refiere, aún a riesgo de simplificar excesivamente lo que es un aproximación decididamente interdisciplinar desde sus inicios, la distribución es la siguiente:

1. Contribuciones referidas a Organizaciones civiles, empresariales y profesionales

\begin{tabular}{|c|c|c|}
\hline Problemática & Análisis de Redes Sociales & N \\
\hline $\begin{array}{c}\text { Desarrollo personal, comunitario y } \\
\text { nacional }\end{array}$ & $\begin{array}{c}\text { Capital y humano } \\
\text { Innovación }\end{array}$ & 11 \\
& $\begin{array}{c}\text { Posiciones y roles } \\
\text { informales } \\
\text { Visualización de grafos }\end{array}$ & \\
\hline
\end{tabular}


2. Contribuciones cuyos referentes remiten al estudio de las prácticas institucionales gubernamentales y contribuciones basadas en estudios sobre transacciones de bienes y servicios

\begin{tabular}{|c|c|c|}
\hline Problemática & Análisis de Redes Sociales & $\mathbf{N}$ \\
\hline $\begin{array}{c}\text { Formulación e implementación de } \\
\text { políticas públicas nacionales }\end{array}$ & $\begin{array}{c}\text { Composición y morfología } \\
\text { estructural }\end{array}$ & 3 \\
& $\begin{array}{c}\text { Medidas de centralidad } \\
\text { Simulación } \\
\text { Visualización de grafos }\end{array}$ & \\
\hline $\begin{array}{c}\text { Sistemas productivos locales y } \\
\text { regionales }\end{array}$ & $\begin{array}{c}\text { Capital social- Embeddedness } \\
\text { Centralidad } \\
\text { Circuitos y jerarquías } \\
\text { Modelización }\end{array}$ & 3 \\
& Visualización de grafos & \\
\hline
\end{tabular}

3. Contribuciones fundadas en el estudio de diversos tipos de comunidades: de emigrantes; virtuales; académicas; étnicas; de profesionales y negocios

\begin{tabular}{|c|c|c|}
\hline Problemática & Análisis de Redes Sociales & $\mathbf{N}$ \\
\hline Procesos de integración social & $\begin{array}{c}\text { Tipología de redes } \\
\text { Micro y macro estructuras } \\
\text { Puentes. Cliques }\end{array}$ & 2 \\
\hline Procesos de enseñanza aprendizaje & Difusión y tecnología & 2 \\
\hline Procesos de producción científica & $\begin{array}{l}\text { Centralidad- Densidad } \\
\text { Coeficiente de Correlación } \\
\text { Visualización de grafos }\end{array}$ & 2 \\
\hline Identidad política y religiosa & $\begin{array}{c}\text { Redes personales } \\
\text { Lazos débiles- fuertes } \\
\text { Técnica de "bola de nieve" }\end{array}$ & 2 \\
\hline $\begin{array}{c}\text { Desarrollo de programas } \\
\text { informáticos para "rentabilizar" las } \\
\text { relaciones }\end{array}$ & $\begin{array}{l}\text { Redes personales y } \\
\text { complejas } \\
\text { Evolución de redes }\end{array}$ & 1 \\
\hline
\end{tabular}

4. Contribuciones cuyos referentes de investigación son los líderes políticos nacionales y los modelos matemáticos

\begin{tabular}{|c|c|c|}
\hline Problemática & Análisis de Redes Sociales & $\mathbf{N}$ \\
\hline Historia de las relaciones de poder & $\begin{array}{c}\text { Centralidad -Cliques } \\
\text { Visualización longitudinal }\end{array}$ & 1 \\
\hline Índices de centralidad & $\begin{array}{c}\text { Modelos estructurales } \\
\text { Centro- Periferia }\end{array}$ & 1 \\
\hline
\end{tabular}

Tabla 4. Distribución de las temáticas 
Desde luego, estas categorías podrían subsumirse en otras mayores. Pero lo que nos interesa es resaltar el carácter aplicado o sustantivo de las contribuciones, más allá del carácter metodológico de las contribuciones que han caracterizado el ARS a nivel internacional hasta hace pocos años.

Pero no sería justo finalizar esta descripción del ARS en Iberoamérica sin resaltar un intangible que entendemos distintivo: la apertura, la informalidad y el dinamismo que lo caracterizan. Hay que decir que estas mismas características son propias de la comunidad internacional, representada por la INSNA. Pocas veces se ha aplicado con más decisión el principio de la fuerza de los vínculos débiles como en la comunidad de rederos internacional. En casa del redero, vínculos débiles primero y fuertes después, aumentando el capital social y disminuyendo los costes de transacción mediante la confianza y transparencia. Hemos demostrado que es posible ganar en conocimiento compartiendo información y trabajando de forma cooperativa. Ahí radica, no nos engañemos ni nos olvidemos, una de las razones del éxito. También, una vez superadas las dificultades del idioma y del aprendizaje de los métodos formales, es necesario colaborar e integrarse con las particularidades y fortalezas a la comunidad internacional.

\section{Destacados en Portorož}

La presentación de los trabajos de nueve investigadores participantes en la IV Mesa Hispanoamericana de la Conferencia del INSNA en Eslovenia ha dado lugar a la publicación del volumen 6 de REDES. Los artículos se centran en los temas y las problemáticas particulares del campo de la economía, de la política y la sociología, pero su proyección metodológica y teórica alcanza a los demás científicos sociales.

Aquí ensayamos un breve avance de sus producciones a modo de versión subtitulada:

Capitalizaciones alternativas para un nuevo concepto profesional. Juan Manuel Larrosa, delimita y caracteriza el ejercicio público y privado de los profesionales del Derecho mostrando los mecanismos actuales que los abogados utilizan para mejorar su desempeño profesional. A través de las 
trayectorias laborales es posible observar los cambios que acontecen desde la visión de los inversores en relacionales sociales.

La revisión de las estrategias seguidas por renombradas firmas legales en Estados Unidos, Canadá y Argentina constituyen una fuente inagotable de elementos para delinear un dominio de entrenamiento, acción y negocios desde la perspectiva de la tradición y el cambio profesional futuro.

Dos conceptos claves alcanzan para introducirnos en el tema: capital humano y capital social. La minuciosa descripción de la dinámica interna de formación de capital humano en las firmas legales se muestra como un proceso complejo que asocia el tipo de formación personal obtenida y los diversos tipos de organizaciones legales.

El capital social se concentra en los beneficios que otorga la disponibilidad de una red de relaciones sociales durables y de los recursos asociados a ellas resultando de interés las instancias intra-organizacionales e interorganizacionales con estructuras de interacción particulares.

Finalmente vislumbrar una carrera laboral prestigiosa parece posible sólo en una estructura social exitosa. Para esto una serie de variables surgen como evidentes al estudio comparado de los casos empíricos. En definitiva: Será Justicia...

Relaciones, sistemas, estructuras: Instituciones, sociedad, territorio. El trabajo de María Semitiel García y Pedro Noguera Méndez se presenta como una aplicación del ARS en el campo de la economía. El planteo de los autores desafía a las habituales explicaciones centradas en los supuestos que sostienen un carácter racionalista del individuo en la toma de decisiones económicas. Mientras que el enfoque de redes se ofrece como un mecanismo explicativo que sitúa a los agentes económicos, la producción y el consumo en niveles de inclusividad basados en las interacciones.

Las nociones de sistemas productivos y capital social se conjugan con las ideas de espacio geográfico e institucional de las actividades económicas. Estas dimensiones localizan las relaciones industriales en el territorio 
español a distintas escalas: local y regional, y contextualizan las interacciones innovadoras a nivel institucional- empresarial.

El análisis de la red de transacciones se basa en datos relacionales provenientes de las tablas de flujos de entrada-salida de grupos empresarios durante dos períodos, 1980 y 1995. Sobre ellos se aplican cálculos de centralidad y de estructura centro-periferia obteniéndose una descripción de las posiciones de las ramas de la industria de acuerdo a la mayor o menor puntuación de coreness o centralidad. Los resultados muestran que en el núcleo de la estructura se hallan las ramas que componen el sistema de producción metal- mecánico y en la periferia quedan las ramas del sistema agro-alimentario de la industria regional vasca.

Visualización en ARS, ¿estética o algoritmo? Alejandro Ruiz León y Jorge Gil- Mendieta observan los cambios en la trayectoria de los políticos mexicanos mediante la visualización comparada de sus redes durante las últimas siete décadas del siglo XX.

En el trabajo de los autores, el diseño de las redes no es simple, ni intuitiva. La representación de la información ha requerido de una enorme base de datos confiables y de una investigación sistemática cuyos crecientes niveles de análisis resultan en las complejas representaciones de las redes. Mediante el soporte del software Pajek se delinean una serie de gráficos donde los nodos activos y sus relaciones son correlacionados por períodos. La consecuencia visual de estos algoritmos es indicada por el color diferencial para los políticos destacados de cada década y las líneas más gruesas indicando la proporción más alta de los lazos que incluyen a algunos personajes en la mayor cantidad de los cliques de pertenencia.

El seguimiento de algunos nodos en las seis redes de políticos entre 1940 y 1990 destaca la participación de 3 ex-presidentes mexicanos. Bajo la forma de los diferentes colores de los nodos se evidencia la salida del escenario político de los líderes revolucionarios y el cambio paulatino y generacional desde 1970, observándose una mayor participación reciente de los actores civiles. 
La escasa cantidad de lazos entre los nodos se grafica conjuntamente con el decrecimiento del grado nodal de los políticos y la distribución de sus relaciones entre los ex presidentes. Esto resulta en una menor integración y cohesión de la red de poder, con un consecuente debilitamiento de la figura presidencial.

Entre el modelo Teórico y el modelo Observado. Una rutina pendular. LoS trabajos orientados a evaluar la fiabilidad de los modelos utilizados en ARS constituyen una labor que es iniciada, alcanzada, probada y vuelta a revisar. Este ritmo explicativo nunca acaba y garantiza el valor predictivo de las herramientas matemáticas. Por esta razón, el trabajo de Ana Salomé García Muñiz el al, propone una reflexión teórica sobre el análisis estructural en redes a través de una crítica a los modelos de centro-periferia.

Estos modelos son indicadores de la densidad o cohesión de las relaciones en una estructura y constituyen una estrategia clásica en varios campos de la ciencia. En este caso, se somete a evaluación el modelo de centroperiferia desarrollado por Borgatti y Everett (1999).

La forma de establecer el centro y periferia de los agentes en una red se logra diferenciando los pares de nodos que tienen mayor grado de centralidad de los que tienen menos. El mecanismo correlaciona una matriz teórica con una observada, pero sin distinguir entre la concordancia o coincidencia de los valores observados y la fuerza de correlación entre ellos.

La propuesta de los autores introduce una alternativa metodológica: la estimación de coeficientes y el análisis de bondad. Los nuevos algoritmos muestran sus ventajas operativas con el objetivo de determinar los índices de centralidad entre los actores, resultando adecuados a la solución del ajuste y acercamiento de las estructuras teóricas a la estructura real.

Redes entre organizaciones $y$ participación comunitaria. La crisis socioeconómica argentina ha sido la antesala de un extenso muestrario de nuevas estrategias de organización y participación comunitaria. Algunas de las experiencias más innovadoras se han dado en los movimientos sociales de base, a través de agrupaciones informales y de pequeñas organizaciones 
comunitarias. Pablo Forni y María Eugenia Longo analizan en el artículo "Las respuestas de los pobres a la crisis" el caso de una agrupación comunitaria del Gran Buenos Aires.

El artículo describe la evolución de una red de organizaciones en una de las áreas más pobres de la capital argentina, destacando el papel del funcionamiento inter-organizacional en la estabilidad, la efectividad y el crecimiento de los movimientos barriales. El estudio de las relaciones entre organizaciones es uno de los temas de más tradición en el análisis de redes sociales. De ese modo, el examen descriptivo en profundidad de las relaciones dentro y fuera del barrio proporciona un conjunto de hipótesis enormemente sugerente para la investigación futura.

El caso de "Comunidad Organizada" muestra la importancia que tienen en los procesos de participación social el liderazgo y la legitimidad de las organizaciones sociales. También ilustra las difíciles relaciones de las redes de organizaciones comunitarias con los actores políticos tradicionales.

Finalmente, pone de manifiesto la relevancia de las relaciones externas a la comunidad, tanto para la consecución de los objetivos pretendidos como para el mantenimiento de las actividades de dichos movimientos sociales.

\section{Una agenda de investigación}

Decíamos a fin de cuentas que había una razón profunda, no coyuntural, de la extensión del ARS: su potencial teórico. Veamos por qué.

Sin pretender ser exhaustivos podemos afirmar que el final del siglo XX ha presenciado el intento de superación de la dicotomía agencia-estructura, micro-macro, objetivismo-subjetivismo. El trabajo de Pierre Bourdieu (1997) o James Coleman (1990) son intentos de superar estas dicotomías. En ambos casos el capital social y las redes sociales son piezas claves de sus propuestas teóricas. Estos intentos de síntesis intentan, a su vez, superar una profunda división epistemológica sobre la posibilidad del conocimiento de la realidad social: la que parte de la conciencia de los actores y de sus acciones (la sociología weberiana) y la que parte de las estructuras independientes de los actores, las instituciones (la sociología 
durkheimniana). No se trata de disquisiciones espúreas. En economía, el triunfo del neoclasicismo ha consagrado en el mismo período una aproximación que parte del individuo y de sus decisiones racionales. Este paradigma, que ha dado innegables frutos, ha tenido que ser corregido sucesivamente por la introducción de los problemas que representan la explicación racional de la acción colectiva, la racionalidad limitada y el papel de las instituciones (neoinstitucionalismo). La Racional Choice Theory, en Ciencia Política, representa igualmente la traslación de las virtudes y dificultades del paradigma neoclásico. De ahí el éxito de las aproximaciones neoinstitucionalistas en el estudio de la agenda política y de las políticas públicas. Granovetter (1985) expresó claramente la situación estratégica de las redes sociales para resolver el problema de desengarzar la acción económica de la relación social. Igualmente, Carlos Lozares (2003) en su artículo de la Revista Redes, sitúa en la interacción el punto de partida del devenir social.

Sin abandonar la Revista Redes nos encontramos con una guía para la acción futura. A partir de la Conferencia de Lille hemos tenido acceso en castellano a contribuciones que sitúan en el campo de lo meso-social, de las mesoestructuras, el lugar estratégico para repensar los problemas sociales y analizar con mayor agudeza los fenómenos sustantivos. Alexis Ferrand nos deslumbró con su artículo sobre las comunidades locales como estructuras meso, Emmanuel Lazega propone superar la propuesta Coleman con su concepto de "nichos sociales", Breiger (2004), uno de los estudiantes de Harrison White, revisita la dualidad de los hechos sociales y la agregación de categorías ... nos encontramos delante de una propuesta teórica de largo alcance, traída por Ainhoa de Federico, la editora de los números correspondientes: situarse en el nivel meso para contribuir a superar las dicotomías teóricas y epistemológicas de las Ciencias Sociales.

En la historia del ARS existe un punto de extraordinario interés: la reelaboración por parte de Harrison White y sus estudiantes (1976 i y ii), de las ideas de Nadel (1957) según el cual la estructura social tenía que ser concebida como una red de roles sociales (o lugares, según la también reciente contribución de Narciso Pizarro, 2004). Es esta noción la que 
permite contemplar al mismo tiempo individuos y estructuras, niveles micro y meso, conciencias y constricciones. De nuevo CONCOR y la idea de equivalencia estructural. Matrices de modo dos la mayor parte de la veces... precisamente las técnicas menos desarrolladas en el ARS en los últimos tiempos.

Ésta es la propuesta: ya sea en el estudio de comunidades, en organizaciones, en redes de acción política, en colegios invisibles... adoptar decididamente un enfoque mesosocial. En ese ámbito podemos recuperar la vieja noción mertoniana (1968) de "teorías de rango intermedio" para explicar los hechos observados sin necesidad de situarse en un paradigma totalmente marxista, neoclásico o weberiano. Este enfoque con la ayuda del ARS nos permitirá hacer contribuciones decisivas a la compresión de los fenómenos sustantivos objeto de estudio, permitiendo recoger la complejidad de las interacciones existentes de una forma totalmente nueva.

Una segunda cuestión que nos atrevemos a recordar: el enfoque de redes no olvida los análisis atributivos clásicos, sino que los incorpora activamente, junto con la visión de los actores implicados. ¿Cómo podría ser de otra forma?

Por último, no hay que olvidar las dificultades con las que nos encontramos. En primer lugar, la falta de una teoría estadística que nos permita obtener muestras representativas de datos relacionales. En segundo lugar, la escasez de test estadísticos aplicables con datos reticulares. Si bien es cierto que los resultados de los análisis se pueden convertir en variables atributivas clásicas, es necesario un desarrollo que permita comparar redes entre sí y en su evolución. Por último, la necesidad de avanzar sobre los métodos de análisis de las matrices de modo 2 y de las aproximaciones basadas en la equivalencia estructural, prácticamente en el mismo lugar que White y sus estudiantes la dejaron.

En cualquier caso, las posibilidades que nos abre el futuro son extraordinarias. Esta Conferencia internacional es un buen ejemplo de todo lo que podemos aprender. Intuimos que podemos entender mejor que 
nuestros mayores el mundo que nos rodea, pero solamente empezando donde los clásicos lo dejaron. No es una visión muy humilde ¿verdad?

\section{Bibliografía}

Boorman, Scott A., and Harrison C. White (1976). "Social Structure from Multiple Networks, II: Role Structure", American Journal of Sociology, 81, 1384-1446.

Bourdieu, Pierre (1977). Outline of a Theory of Practice. New York: Cambridge University Press.

Coleman, J. (1990). Foundations of Social Theory. Cambridge, MA:Harvard University Press.

Ferrand, Alexis (2002). "Las comunidades locales como estructuras meso", REDES- Revista hispana para el análisis de redes sociales, Vol.3,\#4, septnov.

Merton, Robert K. (1968). Social Theory and Social Structure. New York: The Free Press.

Granovetter, Mark S. (1985). "Economic Actions and Social Structure: The Problem of Embeddedness", American Journal of Sociology (481-510).

Lozares, Carlos (2003). "Valores, campos y capitales sociales", REDESRevista hispana para el análisis de redes sociales, Vol.4,\#2, jun.

Nadel, S.F. (1966). Teoría de la estructura social [Theory of social Structure, 1957]. Madrid: Guadarrama-

Narciso Pizarro (2004). "Un nuevo enfoque sobre la equivalencia estructural: lugares y redes de lugares como herramientas para la teoría sociológica", REDES- Revista hispana para el análisis de redes sociales, Vol.5,\#2, ene-feb.

White, Harrison C., Scott A. Boorman \& Ronald L. Breiger: "Social Structure from Multiple Networks, I: Blockmodels of Roles and Positions", American Journal of Sociology, 81, 730-780. 